\title{
Specific targeting of IL-6 signalling pathway: a new way to treat IBD?
}

Atreya R, Mudter J, Finotto S, et al. Blockade of interleukin 6 trans signalling suppresses T-cell resistance against apoptosis in chronic intestinal inflammation: Evidence in Crohn's disease and experimental colitis in vivo. Nat Med 2000;6:583-8.

\begin{abstract}
1
The pro-inflammatory cytokine interleukin (IL) -6 can bind to cells lacking the IL-6 receptor (IL-6R) when it forms a complex with the soluble IL-6R (sIL-6R) (trans signalling). Here, we have assessed the contribution of this system to the increased resistance of mucosal $\mathrm{T}$ cells against apoptosis in Crohn's disease (CD), a chronic inflammatory disease of the gastrointestinal tract. A neutralizing antibody against IL-6R suppressed established experimental colitis in various animal models of CD mediated by type $1 \mathrm{~T}$-helper cells, by inducing apoptosis of lamina propria $\mathrm{T}$ cells. Similarly, specific neutralization of sIL-6R in vivo by a newly designed gp130-Fc fusion protein caused suppression of colitis activity and induction of apoptosis, indicating that sIL-6R prevents mucosal T-cell apoptosis. In patients with $\mathrm{CD}$, mucosal $\mathrm{T}$ cells showed strong evidence for IL- 6 trans signalling, with activation of signal transducer and activator of transcription 3, bcl-2 and bcl-xl. Blockade of IL-6 trans signalling caused T-cell apoptosis, indicating that the IL-6sIL-6R system mediates the resistance of $T$ cells to apoptosis in CD. These data indicate that a pathway of T-cell activation driven by IL-6-sIL-6R contributes to the perpetuation of chronic intestinal inflammation. Specific targeting of this pathway may be a promising new approach for the treatment of CD.
\end{abstract}

Yamamoto M, Yoshizaki K, Kishimoto T, Ito H. IL-6 is required for the development of Th1 cell-mediated murine colitis. F Immunol 2000;164:4878-82.

\section{Abstract 2}

Proinflammatory cytokines have been demonstrated to play a crucial role in the pathogenesis of Crohn's disease (CD). Among those cytokines, strong expression of IL-6 has been repeatedly demonstrated. To examine the role for IL-6 in the pathogenesis of CD, we introduced anti-IL-6R mAb to a murine model of colitis. Colitis was induced in C.B-17-scid mice transferred with CD45RB ${ }^{\text {high }} \mathrm{CD} 4+\mathrm{T}$ cells from $\mathrm{BALB} / \mathrm{c}$ mice. AntiIL-6R mAb or rat IgG was administered weekly after T cell transfer. ICAM-1 and VCAM-1 expression were analyzed by immunohistochemistry. Colonic cytokine expression was determined by RT-PCR. Mice treated with $\mathrm{mAb}$ showed normal growth, whereas controls lost weight. The average colitis score was 0.64 for $\mathrm{mAb}$ treated mice and 1.80 for controls. $T$ cell expansion in treated mice was less remarkable than in the controls. Colonic ICAM-1 and VCAM-1 expression were markedly suppressed by mAb. IFN-, TNF-, and IL-1 mRNA were reduced by the treatment. The results presented here show a crucial role for IL- 6 in the pathogenesis of murine colitis and suggest a therapeutic potential of antiIL-6R mAb for treatment of human CD.

\section{Comment}

More than 200 cytokines have been identified that bind to specific receptors expressed on the surface of the target cells. They are able to trigger intracellular signalling cascades leading to the control of gene expression involved in the cellular response. Although a lot of detailed information on the signalling cascade and effects of specific cytokines on various cells has accumulated over the past few years, our understanding of cytokine functions in vivo remains very poor.

These uncertainties are particularly illustrated by the case of interleukin (IL)-6. The in vivo functions of this cytokine remain debated, IL-6 being considered alternatively as a proor anti-inflammatory cytokine, ${ }^{12}$ or sometimes as a key factor to polarise Th2 cells. ${ }^{3}$ Moreover, the IL- 6 signalling is particular involving a phenomenon called trans signalling. ${ }^{4}$ Briefly, the receptor for IL-6 consists of two subunits: a ligand binding component (IL-6R) and a signal-transducing glycoprotein 130 (gp130) which is a member of the cytokine receptor superfamily (including also IL-11 and the leukaemia inhibitory factor). A soluble form of the ligand specific chain (sIL-6R), when complexed to IL-6, is capable of binding to the membrane bound gp130 and thus can elicit a signal-transduction involving STAT- $3 .^{5}$ This phenomenon called trans signalling introduced a novel aspect of cytokine action (fig 1).

In Crohn's disease (CD), IL-6 is present at high levels in both serum and intestinal tissues. ${ }^{6}$ Increased levels of IL-6R and gp130 expression have been also demonstrated in peripheral lymphocytes of patients with $\mathrm{CD}$ together with an enhancement of serum sIL-6R. ${ }^{7}$ IL-6 signalling playing potentially a crucial role in the pathogenesis of $\mathrm{CD}$, the therapeutic potential of antibodies against IL-6R (antiIL-6R Ab), which block both the transmembrane and soluble forms of IL-6R, has been evaluated. ${ }^{8}$

In the study by Yamamoto et al, colitis was induced by $\mathrm{CD}_{45 \mathrm{RB}^{\text {high }}} \mathrm{CD} 4+\mathrm{T}$ cell transfer in scid mice. ${ }^{8}$ AntiIL-6R Ab was administered intraperitoneally just after $\mathrm{T}$ cell transfer and then weekly for 8 weeks. Anti-IL-6R Ab prevented wasting disease and the development of macroscopic and histological lesions. This treatment suppressed also the massive accumulation of ICAM-1 positive cells in the lamina propria and the expression of ICAM-1 and VCAM-1 by vascular endothelial cells. Expansion of both colonic and splenic transferred CD4+ T cells observed in the recipient untreated scid mice was also reduced as well as the colonic expression of TNF- $\alpha$, IL- $1 \beta$ and IFN- $\gamma$ mRNA without modification for the production of TGF- $\beta$, 


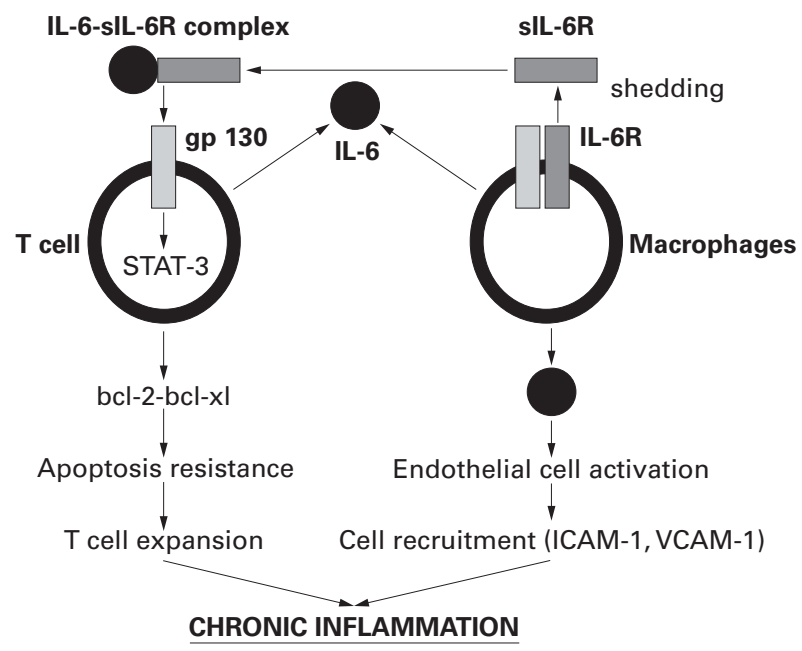

Figure 1 The receptor for IL-6 consists of the ligand binding component (IL-6R) and the signal-transducing gp130. The soluble form of the IL-6R may form a complex with IL-6 having the ability to bind gp130 (trans signaling) expressed by $T$ cells and to activate the anti-apoptotic STAT-3 dependent genes bcl-2 and bcl-xl. In inflammatory diseases, targeting of IL-6 signaling pathway modulates inflammation at least in part through the regulation of $T$ cell expansion and the expression of adhesion molecules.

IL-10 and IL-4 mRNA. This study provides evidence that IL-6 signalling plays a crucial role for the pathogenesis of murine colitis.

The second study published by Atreya et al confirms and extends these results. ${ }^{9}$ The authors showed high production of IL- 6 and sIL-6R by purified lamina propria cells of patients with $\mathrm{CD}$ and $\mathrm{UC}$ together with increased serum levels of IL-6-sIL-6R complexes. In contrast, only a small fraction of $T$ cells in patients with IBD expressed membrane bound IL-6R. However, there was an activation of the IL-6 pathway in these T cells as reflected by an increased expression and nuclear translocation of the IL-6 dependent STAT-3 signalling and a considerable induction of two anti-apoptotic STAT-3 dependent genes namely bcl-2 and bcl-xl. As IL-6 is known to rescue T cells from apoptosis, ${ }^{10}$ the authors next tested a neutralising antibody against human IL-6R which actually induced lamina propria $\mathrm{T}$ cell apoptosis in CD. The next step of the study aimed to evaluate the effects of blocking the IL-6-STAT-3 pathway in three different animal models of colitis associated with increased IL- 6 production and STAT- 3 activation: colitis in $\mathrm{T}$ cell reconstituted scid mice, colitis in IL-10 deficient mice (IL-10-/-) and colitis in mice receiving the hapten reagent trinitrobenzene sulphonic acid (TNBS). In the three models, treatment with anti-IL-6R $\mathrm{Ab}$ suppressed or reduced colitis activity at a similar level to that observed after treatment using antibody against TNF- $\alpha$. Using a newly developed fusion protein gp 130 (gp130-Fc), the authors showed that the therapeutic effect of anti-IL-6R Ab was not due to the blockade of the IL-6 membrane bound receptor but to the sIL-6R.

The therapeutic effects of the anti-IL-6R Ab involved the induction of $\mathrm{T}$ cell apoptosis which occurred exclusively with co-culture of CD4+ T cells and monocytes. As T cells express only gp130 and monocytes are known to produce large quantities of sIL-6R, the candidate molecule for such paracrine interactions between $\mathrm{T}$ cells and monocytes could be the sIL-6R. The role of sIL-6R in the regulation of apoptosis was then clearly demonstrated showing that (1) specific neutralisation of sIL-6R by gp 130-Fc induced apoptosis, (2) treatment with gp130-Fc caused induction of apoptosis similar to that produced by the anti-IL-6R Ab in the colon of mice in vivo, and (3) apoptosis induced by anti-IL-6R Ab can be blocked by hydroxamic acid based metalloprotease inhibitors that inhibit shedding of the IL-6R.

These studies are the first to directly demonstrate an essential pathogenic function for the sIL-6R through IL-6 trans signalling in an inflammatory disease model in vivo. These results suggest that IL- 6 trans signalling may participate in disease perpetuation in IBD by mediating the resistance of lamina propria $\mathrm{T}$ cells against apoptosis. This hypothesis is consistent with recent reports showing that resistance of CD $\mathrm{T}$ cells to multiple apoptotic signals is associated with increase bcl-2 expression, ${ }^{11}$ an antiapoptotic STAT-3 dependent gene. Many questions remain unanswered about the differences in the IL-6 signalling pathway between CD and UC or the benefit in IBD of IL-11 treatment which also uses the common gp130 signal transducer. ${ }^{12}$ However, these data support the hypothesis that physiopathological mechanisms in CD could be mediated by release of sIL-6R by lamina propria macrophages forming complexes with IL-6, having the ability to stimulate gp 130 on the surface of lamina propria $\mathrm{T}$ cells in the gut, and then causing a STAT-3 dependent intestinal $\mathrm{T}$ cell resistance to apoptosis.

In conclusion, specific targeting of the IL-6 signalling pathway may be a new way to treat $\mathrm{CD}$ or inflammatory diseases characterised by increased production of IL- 6 .

P DESREUMAUX

Laboratoire de Recherche sur les Maladies Inflammatoires Intestinales, CHU Lille, France (CRI4U004B)

pdesreumaux@chru-lille.fr

1 Fattori E, Cappelletti $M$, Costa $\mathrm{P}$, et al. Defective inflammatory response in interleukin 6-deficient mice. $\mathcal{F}$ Exp Med 1994;180:1243-50.

2 Aderka D, Le JM, Vilcek J. IL-6 inhibits lipopolysaccharide-induced tumor necrosis factor production in cultured human monocytes, U937 cells, and in mice. F Immunol 1989;143:3517-23.

3 Rincon M, Anguita J, Fikrig E, et al. Interleukin (IL)-6 directs the differentiation of IL-4 producing CD4+ T cells. F Exp Med 1997;185:461-9.

4 Peters M, Müller A, Rose-John S. Interleukin-6 and soluble interleukin-6 receptor: direct stimulation of gp130 and hematopoieisis. Blood 1998;92: receptor:

5 Ohtani T, Ishihara K, Atsumi T, et al. Dissection of signaling cascades through gp130 in vivo: reciprocal roles for STAT3- and SHP2-mediated signals in immune responses. Immunity 2000;12:95-105.

6 Gross V, Andus T, Caesar I, et al. Evidence for continuous stimulation of interleukin-6 production in Crohn's diesase. Gastroenterology 1992;102: 514-9.

7 Mitsutama K, Toyonaga A, Sasaki E, et al. Soluble interleukin-6 receptors in inflammatory bowel disease: relation to circulating interleukin-6. Gut 1995;36:45-9.

8 Yamamoto M, Yoshizaki K, Kishimoto T, et al. IL-6 is required for the development of Th1 cell-mediated murine colitis. F Immunol 2000;164: 4878-82.

9 Atreya R, Mudter J, Finotto S, et al. Blockage of IL-6 trans signaling suppresses T-cell resistance against apoptosis in chronic intestinal inflammation: evidence in Crohn disease and experimental colitis in vivo. inflammation: evidence

10 Teague K, Marrack P, Kappler JW, et al. IL-6 rescues resting mouse T cells from apoptosis. F Immunol 1997;158:5791-6.

11 Ina K, Itoh J, Fukushima K, et al. Resistance of Crohn's disease T cells to multiple apoptotic signals is associated with a bcl-2/bax mucosal imbalance. F Immunol 1999;163:1081-90.

12 Sands BE, Bank S, Sninsky CA, et al. Preliminary evaluation of safety and activity of recombinant human interleukin-11 in patients with active Crohn's disease. Gastroenterology 1999;117:58-64. 